\title{
Herramientas no invasivas de observación en la evaluación de procesos de alfabetización informacional: Uso de Camstudio
}

\author{
Rodrigo Donoso Vegas \\ Universidad Tecnológica Metropolitana - UTEM, Chile \\ Marcelo González Figueroa \\ Universidad de Concepción - UdeC, Chile
}

\section{CASE REPORTS}

\begin{abstract}
Resumen
Se propone el uso de aplicaciones "Screen Video Capture" (SVC) para observar y evaluar competencias de Alfabetización Informacional (ALFIN). La aplicación propuesta es capaz de registrar en video toda la actividad visualizada en la pantalla de un computador. Lo anterior permite la observación "no invasiva" de procesos como la búsqueda, la selección y uso de información. Se presenta una experiencia piloto en la que se utilizó el software de código abierto CamStudio y se orienta en el uso investigativo de ésta aplicación.
\end{abstract}

Palabras clave

Alfabetización informacional ; Metdología de investigación; Técnicas de observación ; Sistemas de observación remota ; Herramientas de observación ; Captura de pantalla de video ; CamStudio

\section{Noninvasive monitoring tools in evaluation of information literacy process: Using Camstudio}

\begin{abstract}
We propose to use application "Screen Video Capture" (SVC) to observe and assess information literacy skills (information literacy). The proposed application is able to record all activity video displayed on a computer screen. This observation allows "non-invasive" as the search process, the selection and use of information. We present a pilot experiment in which we used the open source software CamStudio and focuses on research use of this application.
\end{abstract}

Keywords

Information literacy; Methodology research ; Observation techniques ; Remote observation systems ; Observation tools ; Screen Video Capture ; CamStudio 


\section{Introducción}

Desde que Zurkowski definiera alfabetización informacional (1974) o ALFIN, el concepto ha cobrado un lugar fundamental en el contexto de las competencias requeridas para el desenvolvimiento en ámbitos formativos y laborales en las denominadas sociedades del conocimiento (UNESCO, 2005). Actualmente se entiende como alfabetización informacional al conjunto de habilidades que permite "reconocer cuándo se necesita información y poseer la capacidad de localizar, evaluar y utilizar eficazmente la información requerida” (ACRL, 2000).

Evaluar la ALFIN implica lograr observaciones adecuadas de conductas que indiquen competencia. En el contexto de enseñanza-aprendizaje de la alfabetización informacional la investigación evaluativa se ha centrado en el análisis de "productos" que solicita un tutor (producción de un texto, acierto de búsquedas, etc.) y en menor medida en la observación de los procesos concretos asociados a la búsqueda, la selección y el uso de información. Lindauer indica que los métodos tradicionales de evaluación de la ALFIN se han centrado en la observación de "productos" que son elaborados por los estudiantes (2006).

Centrar la mirada sólo en "productos" podría significar una visión parcial de los fenómenos vinculados a la ALFIN, los que son por definición "procesos complejos", no observables en su totalidad a través de los "productos" que se elaboran. Tras un mismo resultado pueden encontrarse procesos de elaboración muy diversos. En suma, actualmente las técnicas más utilizadas corren el riesgo de producir indicadores que no son adecuados para lo que se desea evaluar.

Por otra parte, la investigación evaluativa de competencias ALFIN utiliza técnicas de observación que en su mayoría presentan altos grados de invasividad, lo que es común a las disciplinas de las ciencias sociales, educativas y de la información. Técnicas como las entrevistas estructuradas y no estructuradas, encuestas o estudios cualitativos, grabaciones de vídeo, la observación directa con presencia del observador-investigador, alteran en general la conducta que se desea observar (Tang et al., 2006).

La estrategia de "observación remota" a través de redes de computadores ha sido una alternativa de observación que presenta ventajas y oportunidades para observar el comportamiento en pantalla de un usuario de la red. Ésta se basa en la observación en tiempo real de la actividad de un grupo de computadores utilizando las herramientas que en general provee un software de administración de red. Para estos fines resultan especialmente útiles los denominados software de "control remoto y gestión de escritorio", como NetSupport School. Estas herramientas diseñadas con fines didácticos posibilitan la observación no invasiva de la actividad visualizada en las pantallas de un grupo de computadores en red.

En el contexto antes señalado, la observación sistemática de registros de video como las que permiten las herramientas de Screen Video Capture, surge como una alternativa para superar los límites de las técnicas más utilizadas de investigación evaluativa, permitiendo de manera simultánea la "observación de procesos" y la "observación no invasiva". Esta modalidad de registro permite registrar procesos continuos de interacción vinculados a cualquier situación de uso de un computador.

Las herramientas denominadas "Screen Video Capture" han sido empleadas con diversos fines, entre ellos la producción de videos instruccionales (Charnigo, 2009), la medición de usabilidad de sitios web (Goodwin, 2005), la enseñanza (Jaki, 2009) y recolección de datos empíricos en el trabajo con software (Thorsteinsson \& Page, 2007). Existiendo una amplia gama de usos posibles aún no explorados.

\section{Software Screen Video Capture}

Los programas de captura de pantalla permiten el registro en archivos de video de toda la actividad visualizada en monitores de computador como el movimiento del mouse, los puntos dónde se realizan clics, entre otros. La oferta de este tipo de sistemas informáticos incluye software libre y de pago. Entre los pagados se encuentra Camtasia y Jing (ambos de TechSmith) y Captivate de Adobe. Camstudio, Wink y el complemento Capture Fox para Firefox destacan a su vez entre los libres. 
Camstudio es un software open source, bajo licencia GPL, disponible para ser utilizado en sistema operativo Windows que permite la grabación de videos en formato AVI y SWF y la captura de secciones completas de la pantalla o partes de ella. Se encuentra actualmente disponible en inglés y alemán.

Camstudio permite la grabación de audio y la personalización de videos con anotaciones y marcas de agua. Cuenta con la posibilidad de ocultar el uso del software mientras éste se encuentra grabando, lo que permite que los usuarios del computador no perciban su funcionamiento. El software puede ser descargado de la página oficial http://camstudio.org/ y su última versión es Camstudio 2.7.

\section{Una experiencia piloto con Camstudio}

A continuación se describe brevemente una experiencia piloto de evaluación de competencias de ALFIN empleando herramientas Screen Video Capture. La actividad se desarrolló con alumnos universitarios de la carrera de sociología de la Universidad Católica de Temuco-Chile en abril del 2011.

La evaluación piloto se desarrolló en la cátedra Introducción a la Sociología para alumnos de primer año. Consistió en una actividad de diagnóstico de habilidades de búsqueda, acceso y uso de información, implementada en un laboratorio de computación.

\subsection{Preparación de laboratorio}

Para la evaluación piloto fue utilizado Camstudio, software usado con estos fines en ámbitos educativos (Jaki, 2009), instrucción de servicios en bibliotecas (Gentle, 2009) y alfabetización informacional (Imler \& Eichelberger, 2011).

Se dispuso de un laboratorio de computación con 30 computadores en red, cada uno con sistema operativo Windows 7, Microsoft Office, procesador Pentium dual core, 3 Gb RAM y 160 Gb de disco duro, además todos los equipos contaron con acceso a internet. Para evitar el registro de información personal de los participantes se bloquearon los accesos a correos electrónicos y a de redes sociales mediante las utilidades de NetSupport School.

Camstudio fue instalado en cada equipo y se activó la función Record para iniciar la grabación. Se utilizó la opción "Full screen" (Region/Full screen), que permite la grabación de toda la actividad que se muestra en pantalla.

En el menú Options (Options/Program Options), se activó la configuración "Minimize program on start recording" y "Hide flashing rectangle during recording". Estas opciones permiten minimizar y ocultar la interfaz del programa durante la grabación evitando la detención por parte del usuario. Las opciones de audio no fueron habilitadas durante el ejercicio.

\subsection{Perfil de los participantes}

Participaron de la actividad 12 hombres y 11 mujeres, con edades que fluctuaban entre los 18 a los 22 años, provenientes de comunas de pequeño tamaño y alto nivel de ruralidad ubicadas en la zona centro-sur de Chile. Sólo ocho alumnos provenían de capitales regionales o provinciales. En relación al acceso a TICs, 17 estudiantes declararon tener computador de uso personal (10 notebook, 2 netbook, 4 PCs), seis no lo tienen y uno no informó al respecto. Los estudiantes declaran utilizar sus computadores en promedio 3,2 horas diarias y 5,4 días a la semana.

\subsection{Productos solicitados}

Los estudiantes recibieron una hoja de instrucciones en que se solicitaban dos productos. Los estudiantes disponían de 30 minutos para realizar ambas actividades. 
Actividad 1: Buscar en el catálogo online de la Biblioteca Universitaria fuentes bibliográficas que permitieran confeccionar una bibliografía sobre un tema libre.

Objetivo: El objetivo de la actividad era observar la usabilidad del catálogo en línea y revisar la capacidad de encontrar información, asimismo observar las palabras claves para operar la búsqueda y la definición de estrategias de búsquedas.

Producto esperado: Documento Word con las referencias bibliográficas de los documentos encontrados.

Actividad 2: Buscar en Internet (buscadores, bases de datos, sitios web) información que permita definir el concepto "Focus Group".

Objetivo: Estas tareas permitían evaluar de manera general los componentes de la Alfabetización Informacional, tales como la selección, evaluación y uso ético de información.

Producto esperado: Documento Word con el registro de una selección de información y la elaboración de una definición.

Ambas tareas se enmarcan en la concepción de la alfabetización basada en destrezas específicas (Bawden, 2002 p.368).

\subsection{Registros obtenidos de la actividad en pantalla}

El tiempo de grabación de los videos abarcó un periodo aproximado de 30 minutos. El formato de grabación utilizado fue AVI. El tiempo de espera para que el equipo del proyecto detuviera las grabaciones generó un aumento en el tamaño de los archivo los que en general superaron los 2Gb. por computador. La última versión de Camstudio permite detener automáticamente la grabación después de un período de tiempo definido por el operador, lo que contribuye a disminuir el tamaño promedio de los videos.

\subsection{Hallazgos más relevantes de la evaluación piloto}

El desarrollo de la actividad y el análisis preliminar de la información permitió evidenciar:

a) En general la grabación de actividades a través de Camstudio resultó eficiente y eficaz para obtener registros continuos (en formato de video), de los procesos de elaboración de productos relacionados con competencias ALFIN. La activación de la función "Hide flashing rectangle during recording" demostró además ser adecuada para lograr una observación no invasiva de las actividades que desarrollaron los participantes en sus computadores.

b) La observación de la usabilidad del catálogo bibliográfico en línea y la capacidad de encontrar información permitió observar el proceso de elaboración de las expresiones de búsqueda, y el uso de estrategias de baja complejidad, registrando además los procesos de ensayo error que los estudiantes realizan. El registro de actividad en pantalla permitió además identificar el tipo de estrategias de navegación o de interrogación que utilizaban los participantes registrando eventos como el número de click, páginas visitadas, errores, etc. La grabación permitió además detectar problemas de usabilidad y/o ubicación de puntos clave del catálogo en línea en el sitio web de la Biblioteca.

c) La observación de tareas relacionadas con los componentes ALFIN, tales como la selección, evaluación y uso ético de información, la experiencia demostró ser útil para analizar las estrategias de búsqueda, uso de información y especialmente para estudiar la producción de textos breves. Al obtener registros dinámicos de los procesos de búsqueda, se registra con detalle el limitado uso de fuentes utilizada por estudiantes, 
además permitir la observación de las técnicas de elaboración de textos basadas en el plagio, la alteración y parafraseo de textos.

\section{Alternativas de análisis de información}

El registro de actividad de pantalla obtenido con un software SVC mostró ser denso y diverso en relación a la cantidad de acciones registradas, lo que permite una variedad de posibles análisis. Lo anterior estará determinado por los objetivos de cada investigación. Sin embargo tanto el análisis cualitativo como el cuantitativo puede basarse en una estrategia de identificación de "citas de video" (pasajes del video que contengan "incidentes" relevantes). Dicha estrategia es común tanto al análisis de contenido cuantitativo (Krippendorf, 1990) y también a las formas de análisis basadas en estrategias cualitativas como la denominada Grounded Theory (Glaser, 1965; Glaser y Strauss, 1965).

Una "cita" en un archivo de audio o sonido puede especificarse como una "marca" en el momento de inicio y de término en que ocurre un incidente a analizar. En términos de contenido corresponde al espacio de tiempo en que ocurre algún incidente relevante para el investigador. Un incidente puede ser un simple "click" o más complejo, como el proceso de elaboración o alteración de una frase.

Cualquiera sea el tipo de análisis que se desee realizar es recomendable contar con la claridad en lo relativo a:

a) identificar cuáles son los eventos o incidentes relevantes en el registro de video y cómo se identifican (cuestión determinada por los objetivos de investigación).

b) determinar el inicio y el final de dicho incidente (la mayor parte de las veces esto es

c) una práctica parcialmente arbitraria pero necesaria para lograr analizar un proceso continuo).

d) marcar y registrar de manera perdurable en el video dichas citas.

e) ser capaz de recuperar las citas y conservarlas como evidencia empírica.

f) clasificar las citas de video para realizar interpretaciones cualitativas, o bien contar el número de incidentes y/o algunas características de los mismos.

Una alternativa de análisis que permite el trabajo con archivos de video son los denominados CAQDAS, entre los que encontramos a Atlas.ti. Este programa permite realizar con eficiencia las actividades de gestión antes señaladas con archivos de video de gran tamaño. La última versión de dicho software cuenta con una versión en español (http://www.atlasti.com/index.html)

\subsection{Problemas éticos}

El uso de herramientas denominadas "Screen Video Capture" está fuertemente limitada por los elementos éticos vinculados al trabajo con poblaciones humanas. Si bien existen diversos criterios a considerar en éste aspecto, los más significativos son el uso o no uso del "consentimiento informado" y el resguardo debido de la confidencialidad de los participantes.

Tomando como criterio normativo al código de ética de la American Sociological Association, se recomienda que sólo en los casos en que las investigaciones se orienten a observar fenómenos de manera no invasiva, no se solicite el consentimiento informado. Es responsabilidad del investigador asegurar en estos casos que las actividades de investigación no impliquen riesgos de ninguna naturaleza para los participantes y analizar si las conductas que se desean observar efectivamente sufren alteraciones al solicitar el consentimiento informado (ASA, 1999, p.12). Piénsese por ejemplo en la observación de conductas no éticas, como el plagio en la elaboración de textos. 
En cualquier otro caso, en que estos supuestos no se cumplan, debe solicitarse como corresponde el consentimiento de los participantes o a los tutores legales de éstos.

Debido al particular tipo de registro de información, el investigador deberá además maximizar la confidencialidad en el registro, almacenamiento y uso posterior de la información. Este principio debe ser preponderante en el caso en que no se considere el consentimiento informado, evitando registrar información personal en cualquier medio, adelantándose a los posibles usos que pudiera tener cualquier forma de registro de información de la investigación (ASA, 1999, pp. 9-11).

\section{Bibliografía}

ACRL. (2000). Information Literacy Competency Standards for Higher Education. Consultado: 9 Dic 2011. Disponible en: http://www.ala.org/acrl/standards/informationliteracycompetency

American Sociological Association. (1999). Code of Ethics and Policies and Procedures of the ASA Committee on Professional Ethics. Washington, DC: American Sociological Association.

Bawden, D. (2002). Revisión de los conceptos de alfabetización informacional y alfabetización digital. Anales de Documentación (5), 361-408.

Charnigo, L. (2009). Lights! Camera! Action! Producing Library Instruction Video Tutorials Using Camtasia Studio. Journal of Library \& Information Services in Distance Learning, 23-30

Glaser, Barney G. (1965). "The Constant Comparative Method of Qualitative Analysis." Social Problems 12:436-445.

Glaser, Barney G. and Anselm L. Strauss. (1967). The discovery of grounded theory: strategies for qualitative research. New York: Aldine.

Gentle, T. (2009). Evaluating CamStudio and Wink screen capture programs for library instruction. Proceedings of an Academic Library Symposium. Consultado: 01 Ago 2013. Disponible en: https://esirc.emporia.edu/handle/123456789/111

Goodwin, S. (2005). Using screen capture software for web site usability and redesign buy-in. Library High Tech, 23 (4), 610-621

Imler, B.; Eichelberger, M. (2011). Using screen capture to study user research behavior. Library High Tech, 29 (3), $446-454$.

Jaki, T. (2009). Recording lectures as a services in a service course. Journal of statistics education. 17 (3).

Krippendorff, Klaus. (1990). Metodología de análisis de contenido: Teoría y práctica. Barcelona: Paidós.

Lindauer, B.G. (2006). Los tres ámbitos de evaluación de la alfabetización informacional. Anales de Documentación (9), 69-81.

Tang, J. et al. (2006). Unobtrusive But Invasive: Using Screen Recording to Collect Field Data on Computer-Mediated Interaction. En: Computer Supported Cooperative Work 2006 Conference. Consultado: 29 Jul, 2013. Disponible en:

http://jameslin.name/research/recent shortcuts/screen recording-cscw2006.pdf

Thorsteinsson, G.; Page, T. (2007). Piloting new ways of collecting empirical data during the fiste project. ICT in education: reflections and perspectives. Bucharest, June 16-17, 2007.

UNESCO. (2005). The Alexandria Proclamation on Information Literacy and Lifelong Learning. Alexandria, Egipto. Consultado: 29 Jul, 2013. Disponible en: http://archive.ifla.org/lll/wsis/BeaconlnfSoc-es.html

Zurkowski, P. (1974). The information service environment relationships and priorities. Reporte, National Commission on libraries and information science, Washington D.C. 


\section{Datos de los autores}

\section{Rodrigo Donoso Vegas}

Bibliotecario Documentalista. Licenciado en Gestión de Información (Universidad Tecnológica Metropolitana). Magister en Comunicación, Educación y Nuevas Tecnologías (Universidad Mayor). Se ha desempeñado principalmente en bibliotecas universitarias en el área de automatización de bibliotecas y como docente en el área de tecnologías de información en la Universidad Tecnológica Metropolitana. Actualmente trabaja en el Instituto Profesional AIEP - Chile

rdonosovegas@gmail.com

\section{Marcelo González Figueroa}

Sociólogo y especialista en asesorías y estudios para organizaciones del sector público y privado. Como docente universitario ha impartido asignaturas de investigación social cualitativa y cuantitativa en las Universidades de Concepción, del Desarrollo, de la Frontera y Católica de Temuco. Actualmente es instructor en los siguientes software de investigación científica: SPSS, STATA, R, Endnote, REDATAM y Atlas.ti.

marcegon@udec.cl

$\begin{array}{ll}\text { Recibido-Received } & : 2013-08-03 \\ \text { Aceptado-Accepted } & : 2013-09-27\end{array}$

(cc) EY New articles in this journal are licensed under a Creative Commons Attribution 3.0 United States License.

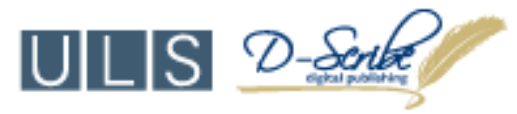

This journal is published by the University Library System of the University of Pittsburgh as part of its D-Scribe Digital Publishing Program and is cosponsored by the University of Pittsburgh Press. 